\title{
Dominance for sn-glycerol-3-phosphate dehydrogenase activity in Drosophila melanogaster: Evidence for differential allelic expression mediated via a trans-acting effect
}

J. B. Gibson, A. V. Wilks, A. Cao and A. L. Freeth

\author{
Department of Population Biology, \\ Research School of Biological Sciences, \\ The Australian National University, \\ P.O. Box 475, \\ Canberra City, A.C.T. 2601, Australia.
}

Two second chromosomes ( $H 31$ and $T 198)$ which when homozygous give relatively low $\alpha$ glycerol-3-phosphate dehydrogenase (GPDH) activity have been isolated from natural populations of Drosophila melanogaster. In heterozygotes between $\mathrm{H31}$ and $\mathrm{T} 198$ and chromosomes bearing standard $G p d h$ alleles there is dominance for GPDH activity and for the amount of GPDH protein. The increased level of activity in the heterozygotes appears to be due to the differential expression of the standard alleles rather than to increased heterodimer activity. Recombination analyses have shown that if the effect is due to a modifier it must be less than $0.04 \mathrm{cM}$ from the $G$ pdh locus.

\section{INTRODUCTION}

In diploid species genetically determined electrophoretic enzyme variants at structural gene loci are invariably codominant. Heterozygotes for alleles which encode electrophoretically distinct proteins are expected to produce both forms and, if the enzyme product is a dimer, hybrid enzyme molecules. If the enzyme products of two distinguishable alleles differ in activity the heterozygotes are expected to be of intermediate activity.

These expectations have usually been fulfilled in electrophoretic surveys of a wide range of animals and plants. The majority of exceptions come from studies of inter-specific hybrids in which there is preferential gene expression of one homologous gene over the other (e.g., CastroSierra and Ohno, 1968; Wright and Moyer, 1966; Scholl and Anders 1973; Herrera, 1979; Whitt, 1981; Dickinson, Rowan and Brennan, 1984). Within species exceptions are much rarer, although differential allele expression exists for alcohol dehydrogenase (Schwartz, 1971) and alphaamylase (Chao and Scandalios, 1971) in maize, 6-phosphoglucose dehydrogenase in carp (Klose and Wolf, 1970), peroxidase in rice (Endo, 1971) and for xanthine dehydrogenase (McCarron,
O'Donnell, Chovnick, Bhullar, Hewitt and Candido, 1979) and ribosomal RNA genes (Procunier and Tartof, 1978) in Drosophila melanogaster. In the case of alcohol dehydrogenase in maize and xanthine dehydrogenase and the ribosomal genes in $D$. melanogaster detailed genetic analyses show that the differential expression is due to tightly linked cis-acting modifiers. (Schwartz, 1971; Procunier and Tartof, 1978; and McCarron et al., 1979).

We describe in this paper a further example in $D$. melanogaster in which functional electrophoretic variants of soluble sn-glycerol-3-phosphate dehydrogenase (sn-glycerol-phosphate; $\mathrm{NAD}^{+}$oxidoreductase; EC 1.1.1.8; GPDH) are differentially expressed in heterozygotes. The regulatory factor is trans-acting and gives rise to dominance in the steady state level of GPDH in heterozygotes (Gibson et al., 1986).

\section{MATERIALS AND METHODS}

The sn-glycerol-3-phosphate dehydrogenase $(G p d h)$ electrophoretic variants, $F$ and $S$, used as standards in these experiments were extracted from flies collected from a natural population of $D$. 
melanogaster at Cygnet (Tasmania). In addition, two putative $G p d h$ alleles with unusual properties, $T 198$ and $H 31$, were extracted from the Tasmanian populations Tamar and Huonville respectively. In all extractions $\mathrm{C} y 0$ and TM6 were used as second and third chromosome balancers (Lindsley and Grell, 1968) and in each stock the third chromosomes were derived from the same populations as the second chromosomes. Gpdh variants with similar properties to $T 198$ and $H 31$ have been isolated from other populations to be described elsewhere.

The Cygnet and Huonville populations are about $10 \mathrm{~km}$ apart in the south of the island and the flies were breeding in waste apple pressings. Tamar is $300 \mathrm{~km}$ to the north and there the flies were breeding in discarded grape pressings.

$T 198$ and $H 31$ (and the other variants with similar properties) were identified in experiments in which up to 300 wild caught male flies from each of the three Tasmanian populations were mated in turn, in single pair cultures, to females homozygous for either $F$ or $S$ standard alleles (O'Brien and MacIntyre, 1970), which were derived from the previous year's collection of flies at Cygnet. The male parents were electrophoretically typed for GPDH phenotypes and the progenies from male $F / S$ heterozygotes discarded. Progenies from cultures where the male parent was homozygous for either $F$ or $S$ were scored for GPDH phenotypes. A number of pairs of unexpected progenies were obtained in which, for example, both $F$ and $S$ homozygotes occurred in one set of progeny and only $F$ homozygotes in the other progeny from the same male. These were explicable if the male parent was heterozygous for a null activity allele at the $G p d h$ locus. Extraction of the relevant second chromosomes showed this to be the correct explanation (Freeth and Gibson, 1985). However, other progenies were unusual in that putative heterozygotes exhibited abnormal electrophoretic phenotypes. A number of second chromosomes giving rise to these variant phenotypes were extracted and made homozygous; these included $T 198$ and $H 31$ which were chosen for further analysis as they differed from each other in electrophoretic mobility. In the experiments described here, the designations $T 198$ and $H 31$ represent second chromosomes containing these putative $G p d h$ alleles.

A number of laboratory stocks were also utilised; $D f(2 L) G d h A$ is an X-ray induced deficiency for clot $(c l)$ and $G p d h$ (Grell, 1967) which extends from 25D7-E1 to 26A8-9 covering about 5cM (Kotarski et al., 1983). Stocks homozygous for $c l . G p d h^{S} . s p d^{f g}$. and $c l . G p d h^{F} . s p d^{f g}$. were kindly provided by Ross MacIntyre.

The GPDH isozymes and their "Fast" and "Slow" electrophoretic variants were identified after electrophoresis of homogenates of single flies on cellulose acetate membranes. The membranes were soaked in running buffer for 1 hour before use. The running buffer contained Tris $(126 \mathrm{mM})$, citric acid (42 mM) and EDTA-di $\mathrm{Na}(9.26 \mathrm{mM})$, $\mathrm{pH} 6 \cdot 6$. Electrophoresis was carried out for 1 hour and the membranes were stained for GPDH activity in $100 \mathrm{mM}$ Tris/ $\mathrm{HCl}(\mathrm{pH} \mathrm{8.6)}$ solution containing DL- $\alpha$-glycerophosphate, NAD, MTT, PMS and EDTA (di-sodium salt).

Adult $D$. melanogaster homozygous for either the $F$ or $S G p d h$ variants exhibit three bands of activity, GPDH-1, GPDH-2 and GPDH-3, ordered in decreasing mobility towards the anode on cellulose acetate membranes. All three isozymes are encoded by the same structural gene (localised to the cytogenetic region 25F-26B), but GPDH-3 is derived from GPDH-1 by a proteolytic cleavage of three amino acids at the $\mathrm{COOH}$-terminus (Niesel et al., 1980; 1982). The minor isozyme, GPDH-2, may arise as a hybrid molecule in tissues where GPDH-1 and GPDH-3 are synthesised (Bewley, Rawls and Lucchesi, 1974). Only GPDH3 is present in third instar larvae. GPDH is a dimer with a sub-unit molecular weight of 32,000 daltons and heterodimers are formed in $F / S$ heterozygotes (Niesel et al., 1980).

Stain intensities of the GPDH isozymes after electrophoresis were measured with a Quick Scan integrating densitometer (Helena Laboratories). The electrophoretic techniques, sample concentrations and staining times were standardised to give a linear relationship between the intensity of the stain and the enzyme concentration in the applied sample (correlation coefficient, 0.97).

For GPDH activity measurements 10 five to six-day old male flies were weighed and ground up at $10 \mathrm{mg} / \mathrm{ml}$ buffer $(0.1 \mathrm{M}$ potassium phosphate, $\mathrm{pH} 7 \cdot 1$ containing $10 \mathrm{mM}$ EDTA disodium, and $1.05 \mathrm{mM}$ dithiothreitol). The homogenates were centrifuged at $17,000 \mathrm{~g}$ for $40 \mathrm{~min}$ at $0^{\circ} \mathrm{C}$. The clear supernatant provided a crude extract. Enzyme assays of the reverse reaction were carried out with the methods and buffers described by Bewley, Rawls and Lucchesi (1974). A unit of enzyme activity is defined as $1.0 \times 10^{-4}$ mole of $\mathrm{NAD}^{+}$reduced per min.

The flies for enzyme assays were reared in 4 " vials with $20 \mathrm{ml}$ of medium containing per litre, $10 \mathrm{gm}$ agar, $15 \mathrm{gm}$ sucrose, $35 \mathrm{gm}$ dried yeast, 
40 gm malt powder, $30 \mathrm{ml}$ corn syrup, $10 \mathrm{gm}$ high protein powder and $4.5 \mathrm{ml}$ proprionic acid.

The amount of GPDH protein was assayed by radial immunodiffusion using the method of Lewis and Gibson (1978). Anti-sera prepared in goats to purified GPDH was a gift from David Sullivan.

\section{RESULTS}

\section{(i) Electrophoretic phenotypes}

The electrophoretic phenotypes of the $F / F$ and $S / S$ controls, the homozygous $T 198$ and H31 strains and the four heterozygotes between these four lines are shown in fig. 1. Densitometer scans of typical electrophoretic patterns from each of the eight genotypes are shown in fig. 2 . These figs show that the $T 198$ homozygotes are electrophoretically indistinguishable from the $S / S$ control, while $H 31$ homozygotes have an identical phenotype to that of the $F / F$ control. In each homozygote there are three bands of activity (GPDH-F1, 2 and 3 or GPDH-S1, 2 and 3) with the middle band (F2 or S2) being the weakest. The $F / S$ heterozygotes give five separate bands of activity. The strongest staining bands are in the positions corresponding to S1 or F3 and F2, which contain heterodimers as well as parental enzyme. The heterozygote $T 198 / H 31$ has the same pattern with five bands of activity. Heterozygotes between $H 31$ and $S$ also have five bands of activity but the pattern is distinguishable from that of the usual heterozygote because the strongest staining band is the third from the cathode, i.e. in position S1 or F3. In marked contrast is the pattern shown by the heterozygote between $T 198$ and $F$. No band is detectable in position S3 (which should have the S3 band of T198) and the S2 band is weak. The strongest band is in the position of F1 which is derived from the $F$ allele.

Scans of 12 electrophoretic separations of each genotype were quantified and the results (table 1) confirm those derived from the visual appearance of the zymograms. There is no significant difference in the relative band intensities between $F / S$ and $H 31 / T 198$ heterozygotes $\left(\chi_{4}^{2}=6 \cdot 69, p>0 \cdot 1\right)$,

Figure 1 Electrophoretic phenotypes of GPDH variants on cellulose acetate membranes: (from left to right): $G p d h^{F} / G p d h^{F}$, $G p d h^{S} / G p d h^{S}, G p d h^{H 31} / G p d h^{H 31}, G p d h^{T 198} / G p d h^{T 198}, G p d h^{F} / G p d h^{S}, G p d h^{F} / G p d h^{T 198}, G p d h^{S} / G p d h^{H 31}$, $G p d h^{T 198} / G p d h^{H 31}$. 
(a)

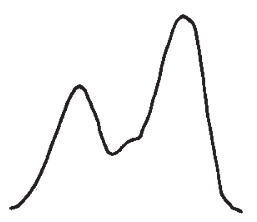

(b)

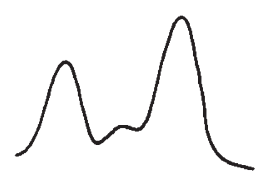

(c)

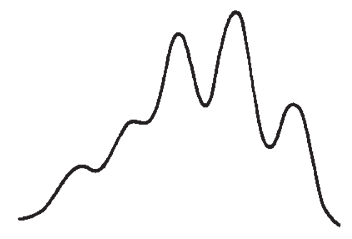

(d)

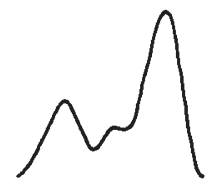

(e)

(f)

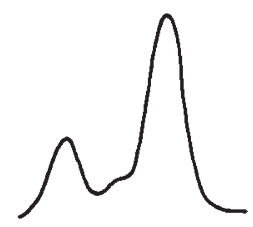

(g)
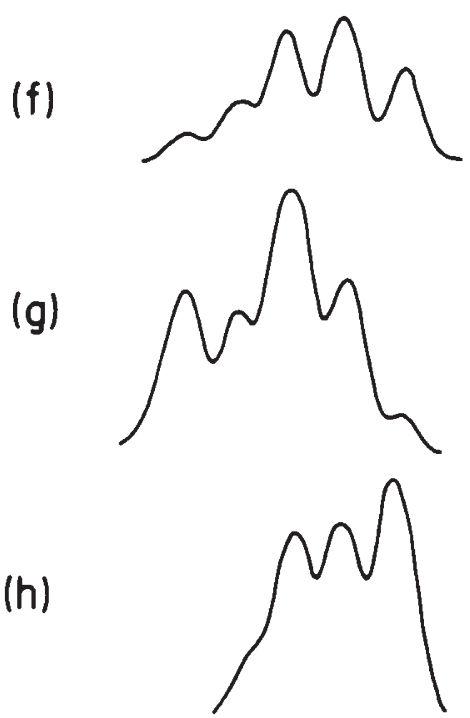

Figure 2 Densitometer scans of GPDH phenotypes after electrophoresis (Note order of genotypes differs from that in fig. 1.) From left to right the five separate peaks are in the positions of bands $\mathrm{S} 3, \mathrm{~S} 2, \mathrm{~S} 1$ or F3, F2 and F1. In normal heterozygotes (c) heterodimer is present in all bands except S3 and F1. (a) $G p d h^{s} / G p d h^{s}$, (b) $G p d h^{F} / G p d h^{F}$, (c) $G p d h^{F} / G p d h^{S}$, (d) $G p d h^{T 198} / G p d h^{T 198}$, (e) $G p d h^{H 31} /$ $G p d h^{H 31}$, (f) $G p d h^{T 198} / G p d h^{H 31}$, (g) $G_{p d h}{ }^{S} / G p d h^{H 31}$, (h) $G p d h^{F} / G_{p d h^{T 198}}$ whereas $H 31 / S$ and $T 198 / F$ differ significantly from each other $\left(\chi_{4}^{2}=253 \cdot 1, p<0 \cdot 001\right)$ and from the $H 31 / T 198$ or $F / S$ heterozygotes $\left(\chi_{4}^{2}=294 \cdot 41\right.$, $p<0 \cdot 001$, and $\chi_{4}^{2}=382 \cdot 56, p<0 \cdot 001$, respectively). Thus three types of GPDH pattern for heterozygotes are clearly distinguishable after electrophoresis even though homozygotes for $T 198$ and $H 31$ have the same patterns as the $F / F$ and $S / S$ controls.

\section{(ii) GPDH activities and amount of protein}

The level of GPDH activity in each of the homozygotes $T 198 / T 198$ and $H 31 / H 31$ is about half the activity of either the $F / F$ or $S / S$ controls (table 2 ) which do not differ significantly from each other in activity. The GPDH activity in the $F / S$ or $T 198 / H 31$ heterozygotes does not differ significantly from their respective mid-parental values $\left(t_{(3)}=1 \cdot 7, p>0.1, t_{(3)}=0.42, p>0.5\right.$ respectively $)$.

However in heterozygotes between $H 31$ or $T 198$ and either of the controls $F$ or $S, G P D H$ activity is significantly higher than the respective mid-parental values $\left(H 31 / F t_{(3)}=13.7, p<0.001\right.$, $H 31 / S t_{(3)}=8.9, p<0.01, T 198 / F t_{(3)}=4.6, p<$ 0.05 and $\left.T 198 / S t_{(3)}=4 \cdot 3, p<0.05\right)$. In each of these four heterozygotes the mean activity was not significantly different to the level of the parent with the highest activity, i.e. the $F / F$ or $S / S$ controls. In all genotypes reciprocal crosses gave similar results (Gibson et al., 1985). Thus the expression of GPDH activity in these heterozygotes exhibits dominance.

To test the generality of the dominance effect we investigated GPDH activity in heterozygotes between $H 31$ and the marker chromosome $c l . G p d h^{s} . s d^{f g}$. Heterozygotes between $H 31$ and cl.Gpdh ${ }^{S}$. spd $^{f g}$ had a mean GPDH activity of $352 \pm$ $10 \cdot 3$, compared with $342 \pm 11 \cdot 8$ for $c l . G p d h^{s} \cdot s p d^{f g}$ homozygotes. The electrophoretic phenotype of the heterozygote on cellulose acetate membranes was characteristic of $\mathrm{H} 31 / \mathrm{S}$.

Assays of flies heterozygous for $F, S, T 198$ or $H 31$ with $D f(2 L) G d h A$ show that in each genotype the level of GPDH activity is about half that of the diploid level (table 3).

Over all genotypes there was a correlation of 0.95 between the amount of GPDH protein measured by radial immunodiffusion and GPDH activity. Heterozygotes between $T 198$ or $H 31$ and $F$ or $S$ had the same amount of GPDH protein as $F / F$ or $S / S$ homozygotes (table 2). Heterozygotes between $T 198, H 31, F$ or $S$ and $D f(2 L) G d h A$ had half the amount of GPDH protein as their respective diploid levels (table 3 ). 
Table 1 Mean relative percentage activity in each band ( \pm standard errors) calculated from the areas under the curves in the densitometer scans

\begin{tabular}{|c|c|c|c|c|c|c|}
\hline \multirow{2}{*}{$\begin{array}{l}\text { Gpdh } \\
\text { genotype }\end{array}$} & \multirow{2}{*}{$\begin{array}{l}\text { No. of } \\
\text { samples }\end{array}$} & \multicolumn{5}{|c|}{ Band of GPDH activity } \\
\hline & & S3 & $\mathrm{S} 2$ & $\mathrm{~S} 1$ or $\mathrm{F} 3$ & F2 & $\mathrm{F} 1$ \\
\hline$S / S$ & 10 & $25 \cdot 7 \pm 1 \cdot 9$ & $8 \cdot 2 \pm 0 \cdot 6$ & $66 \cdot 0 \pm 2 \cdot 3$ & & \\
\hline$F / F$ & 10 & & & $34 \cdot 8 \pm 0 \cdot 8$ & $10 \cdot 5 \pm 0 \cdot 6$ & $54 \cdot 7 \pm 1 \cdot 2$ \\
\hline$F / S$ & 10 & $6 \cdot 9 \pm 0.3$ & $13 \cdot 4 \pm 0 \cdot 6$ & $28 \cdot 0 \pm 0 \cdot 4$ & $32 \cdot 5 \pm 0 \cdot 5$ & $19 \cdot 1 \pm 0 \cdot 7$ \\
\hline$T 198 / T 198$ & 8 & $17 \cdot 9 \pm 1 \cdot 9$ & $9 \cdot 0 \pm 1 \cdot 3$ & $73 \cdot 1 \pm 3 \cdot 1$ & & \\
\hline$H 31 / H 31$ & 12 & & & $29 \cdot 0 \pm 1 \cdot 3$ & $12 \cdot 2 \pm 0 \cdot 8$ & $58 \cdot 8 \pm 1 \cdot 9$ \\
\hline$H 31 / T 198$ & 19 & $5.7 \pm 0.6$ & $12 \cdot 4 \pm 0 \cdot 5$ & $29 \cdot 1 \pm 0 \cdot 4$ & $35 \cdot 2 \pm 0 \cdot 4$ & $17 \cdot 6 \pm 0 \cdot 3$ \\
\hline$S / H 31$ & 16 & $23 \cdot 8 \pm 0 \cdot 5$ & $14 \cdot 3 \pm 0 \cdot 2$ & $39 \cdot 2 \pm 0 \cdot 4$ & $20 \cdot 4 \pm 0 \cdot 3$ & $2 \cdot 3 \pm 0 \cdot 02$ \\
\hline$F / T 198$ & 20 & none detected & $6 \cdot 6 \pm 0 \cdot 4$ & $29 \cdot 1 \pm 0 \cdot 7$ & $26 \cdot 9 \pm 0 \cdot 3$ & $37 \cdot 4 \pm 0 \cdot 9$ \\
\hline
\end{tabular}

Table 2 sn-Glycerol-3-phosphate dehydrogenase activities ( \pm standard errors) and the amount of CRM relative to that observed in the $S / S$ homozygote. Each value is the mean obtained in assays of extracts from four separately prepared cultures. The expected activities given are those if heterozygotes are exactly intermediate between the two parental strains

\begin{tabular}{|c|c|c|c|}
\hline $\begin{array}{l}\text { Gpdh } \\
\text { genotype }\end{array}$ & $\begin{array}{l}\text { GPDH activity } \\
\text { ( } \pm \text { standard error) }\end{array}$ & $\begin{array}{l}\text { Expected } \\
\text { GPDH } \\
\text { activity }\end{array}$ & $\begin{array}{l}\text { Amount } \\
\text { of } \\
\text { CRM }\end{array}$ \\
\hline \multicolumn{4}{|l|}{ Controls } \\
\hline$F / F$ & $416 \cdot 2 \pm 18 \cdot 1$ & - & $1 \cdot 0$ \\
\hline$S / S$ & $418 \cdot 3 \pm 6 \cdot 3$ & - & $1 \cdot 0$ \\
\hline$F / S \dagger$ & $437 \cdot 0 \pm 11 \cdot 6$ & $417 \cdot 2$ & $1 \cdot 04$ \\
\hline$H 31 / H 31$ & $184 \cdot 9 \pm 5 \cdot 2$ & - & $0.45^{*}$ \\
\hline$T 198 / T 198$ & $179 \cdot 9 \pm 5 \cdot 9$ & - & $0.44^{*}$ \\
\hline T198/H31† & $179 \cdot 5 \pm 7 \cdot 0$ & $182 \cdot 4$ & $0.43^{*}$ \\
\hline$H 31 / F \dagger$ & $447 \cdot 9 \pm 10 \cdot 7$ & $300 \cdot 6$ & $1 \cdot 1$ \\
\hline$H 31 / S \dagger$ & $421 \cdot 1 \pm 13 \cdot 4$ & $301 \cdot 6$ & $1 \cdot 0$ \\
\hline$T 198 / F \dagger$ & $382 \cdot 4 \pm 18 \cdot 4$ & $298 \cdot 0$ & 0.96 \\
\hline$T 198 / S \dagger$ & $384 \cdot 4 \pm 19 \cdot 9$ & $299 \cdot 1$ & 0.94 \\
\hline
\end{tabular}

$\dagger$ Reciprocal crosses gave similar results.

* Significantly different to standard $S / S, p<0.001$.

Table 3 sn-Glycerol-3-phosphate dehydrogenase activity ( \pm standard errors) and the amount of CRM relative to that observed in the $S / S$ homozygotes, in flies heterozygous for $D f(2 L) G d h A$. Each value is the mean of assays of extracts of flies from four separate cultures

\begin{tabular}{|c|c|c|c|}
\hline $\begin{array}{l}\text { Gpdh } \\
\text { genotype }\end{array}$ & $\begin{array}{l}\text { GPDH } \\
\text { activity }\end{array}$ & $\begin{array}{l}\text { Expected } \\
\text { half } \\
\text { diploid } \\
\text { value }\end{array}$ & $\begin{array}{l}\text { Amount } \\
\text { of } \\
\text { CRM }\end{array}$ \\
\hline$F^{\prime} / D f(2 L) G d h A$ & $223 \cdot 6 \pm 8 \cdot 1$ & $208 \cdot 1$ & $0 \cdot 58$ \\
\hline$S / D f(2 L) G d h A$ & $230 \cdot 7 \pm 10 \cdot 4$ & $209 \cdot 1$ & 0.55 \\
\hline$H 31 / D f(2 L) G d h A$ & $97 \cdot 4 \pm 8 \cdot 4$ & $92 \cdot 4$ & $0 \cdot 21^{*}$ \\
\hline$T 198 / D f(2 L) G d h A$ & $87.4 \pm 5.8$ & $89 \cdot 9$ & $0 \cdot 21^{*}$ \\
\hline
\end{tabular}

* Significantly different to half the diploid CRM level, $p<$ 0.001 .

\section{(iii) Larval patterns}

The electrophoretic phenotypes of H31 and T198 in heterozygotes with $S$ or $F$ are essentially the same in 3rd instar larvae as in adults (although in larvae only the GPDH-3 isozyme is present). Despite this simpler pattern we have been unable to obtain band separation as clear as that for adults. In 3rd instar larvae GPDH activity exhibits dominance in heterozygotes between the standard alleles and either $H 31$ or $T 198$, a finding which will be reported more fully elsewhere.

\section{(iv) Are T198 and H31 Alleles at the Gpdh locus?}

In the breeding programme used to extract the second chromosomes bearing $\mathrm{T} 198$ and $\mathrm{H} 31$ the variants behaved as alleles at the Gpdh locus. If however the differential expression of, for example $T 198$, is due to a modifier linked to the $G p d h$ locus (i.e., $m S$ ) then it would be possible by recombination to separate the modifier from the $S$ allele. To test this possibility we investigated the electrophoretic phenotypes of recombinant progeny produced in the crosses T198/cl.F.spd $\$ \times$ cl.F.spd/cl.F.spd $\delta$ and H31/cl.S.spd $\$ \times$ cl.S.spd/ cl.S.spd $\delta$. In 403 recombinants we have not been able to separate any modifier effect from either $T 198$ or $H 31$ and produce a normal $F / S$ heterozygote (table 4). As the map distance $c l-G p d h$ is $1.67 \mathrm{cM}$ and $G p d h$-spd $d^{f g}$ is $4.65 \mathrm{cM}$ any modifier of $T 198$ must be less than $0.027 \mathrm{cM}$, and of $H 31$ less than $0.037 \mathrm{cM}$, from the $G p d h^{+}$locus.

\section{DISCUSSION}

The putative alleles $H 31$ and $T 198$ are distinguishable from the standard $F$ and $S$ alleles by their 
Table 4 GPDH phenotypes of recombinant genotypes in the $c l-s p d^{f g}$ region

\begin{tabular}{llll}
\hline & \multicolumn{3}{c}{ Marker phenotype } \\
\cline { 2 - 4 } Chromosome & $\begin{array}{l}\text { GPDH } \\
\text { phenotype }\end{array}$ & $c^{+} . s p d^{f g}$ & cl.spd $^{f g^{+}}$ \\
\hline$H 31$ & $S / S$ & 25 & 76 \\
& $H 31 / S$ & 52 & 20 \\
$T 198$ & $F / S$ & - & - \\
& $F / F$ & 17 & 83 \\
& $T 198 / F$ & 84 & 46 \\
\hline
\end{tabular}

relatively low GPDH activities in homozygotes and by their effect on GPDH activities and electrophoretic phenotypes in heterozygotes with the normal alleles. Homozygotes for the chromosomes bearing $H 31$ and $T 198$ produce electrophoretic phenotypes on cellulose acetate membranes indistinguishable from those of standard $F$ or $S$ homozygotes respectively, and heterozygotes between $T 198$ and $H 31$ have the same phenotype as $F / S$ heterozygotes. Yet heterozygotes $F / T 198$ and $S / H 31$ have phenotypes that differ from each other and from $F / S$. Although the homozygotes $T 198 / T 198$ and $H 31 / H 31$ each have about half of the GPDH activity of the standard homozygotes, the heterozygotes $T 198 / F$ or $S$ and $H 31 / S$ or $F$, have similar activities to the standard homozygotes.

The increased activity of the heterozygotes between $T 198$ or $H 31$ and the standard chromosomes is paralleled by an increase in the amount of GPDH protein (CRM) compared to the midparental value. These data, and the electrophoretic phenotypes, argue against the idea that the dominance effect is due either to the hybrid enzyme having a higher catalytic efficiency than the homodimers (Fincham, 1966) or to increased amounts of heterodimer. The question thus arises as to whether the expression of both alleles is increased in heterozygotes. The densitometer scans show that in $F / T 198$ the $\mathrm{S} 3$ band derived from
$T 198$ is too faint to detect and that the heaviest staining band is in the $\mathrm{F} 1$ position derived from $F$. In $H 31 / S$ heterozygotes there is heavy staining in the $\mathrm{S} 3$ and $\mathrm{S} 1$ or $\mathrm{F} 3$ band positions. Based on the average percentage composition of the $\mathrm{F} 1$ band of $F / F$ and the $\mathrm{S} 1$ band of $T 198 / T 198$, the $\mathrm{F}_{1}$ band of $F / T 198$ would be expected to have about 24 per cent of the total activity of $F / T 198$, whereas it has 37 per cent (table 1). Similar calculations show that the $\mathrm{S}_{3}$ band of the $F / T 198$ heterozygotes was expected to have 1.2 per cent total activity, whereas no band was detected. Also, in the $S / H 31$ heterozygotes the $\mathrm{S}_{3}$ band was expected to be 12 per cent of the total activity, whereas it accounted for 22.1 per cent, and the $F_{1}$ band was 3.0 per cent, whereas 4.9 per cent was expected. Thus these data suggest that in the $F / 198$ and $S / H 31$ heterozygotes the GPDH-F or GPDH-S enzyme, respectively, is present at higher levels than expected. Assuming that all the activity above mid-parental level in, for example, $F / T 198$ is due to the presence of more product of the $F$ allele (and that the product will be present as GPDH-1, GPDH-2 and GPDH-3 in the same proportions as in $F / F$ ) the expected proportions of activity in each of the five bands is given in table 5. These data and those derived in a similar manner for $S / H 31$, show that the observed and expected proportions are not significantly different $\left(F / T 198, \chi_{4}^{2}=2.65, p>\right.$ $\left.0.05, S / H 31, \chi_{4}^{2}=2 \cdot 74, p>0.05\right)$.

We have quantified the electrophoretic phenotypes of $F / H 31$ and $S / T 198$ heterozygotes after the cellulose acetate membranes have been held in a water bath for $20 \mathrm{~min}$. at $50^{\circ} \mathrm{C}$ prior to staining. This removes the heat labile isozymes GPDH-2 and GPDH-3 (Bewley et al., 1974; Bewley, 1978) so that the heterozygotes are expected to have three bands of activity derived from GPDH-1. Fig. 3 and table 6 show that in $S / H 31$ and $F / T 198$ the homodimer bands, S1 or F1 respectively, are twice as intense as the heterodimer bands. In each genotype the relative proportions of the bands suggest that the enzyme produced by the normal allele is present at more than four times

Table 5 The expected percentage activities in heterozygotes $F / T 198$ and $S / H 31$ based on the data in tables 1 and 2 and assuming that the increased activity in the heterozygotes is solely due to the $F$ or $S$ alleles

\begin{tabular}{lccccc}
\hline \multirow{2}{*}{$\begin{array}{l}\text { Gpdh } \\
\text { genotype }\end{array}$} & \multicolumn{5}{c}{ Band of GPDH activity } \\
\cline { 2 - 6 } & S3 & S2 & S1 or F3 & F2 & F1 \\
\hline$F / T 198$ & 0.6 & 7.8 & 30.8 & 30.6 & 26.9 \\
$S / H 31$ & 14.9 & 14.4 & 46.7 & 21.4 & 2.6 \\
\hline
\end{tabular}




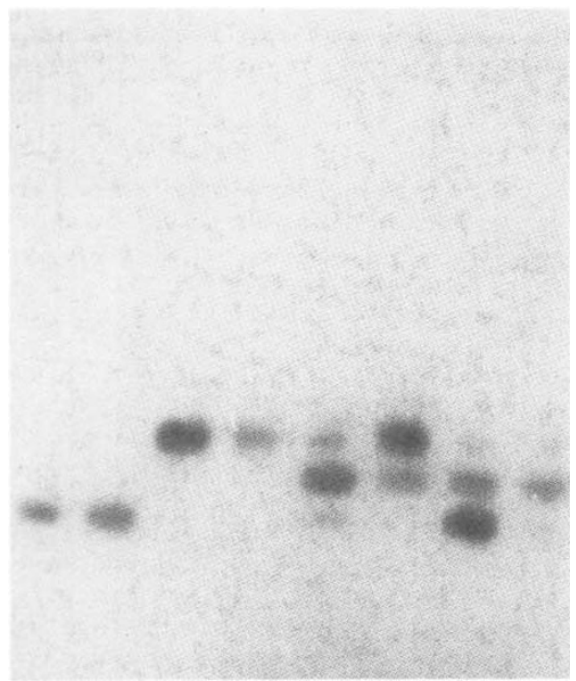

Figure 3 GPDH electrophoretic phenotypes after cellulose acetate membranes have been held in a water bath at $50^{\circ} \mathrm{C}$ for $20 \mathrm{~min}$ prior to staining: (from left to right): $G p d h^{F} / G p d h^{F}, \quad G p d h^{H 31} / G p d h^{H 31}, \quad G p d h^{S} / G p d h^{S}$, $G p d h^{T 198} / G p d h^{T 198}, G p d h^{F} / G p d h^{S}, G p d h^{S} / G p d h^{H 31}$, $G p d h^{F} / G p d h^{T 198}, G p d h^{H 31} / G p d h^{T 198}$.

Table 6 Mean percentage activity remaining in each band ( \pm standard error) after cellulose acetate membranes were held in a water bath at $50^{\circ} \mathrm{C}$ for $20 \mathrm{~min}$ prior to staining for GPDH

\begin{tabular}{lllll}
\hline \multirow{2}{*}{$\begin{array}{l}\text { Gpdh } \\
\text { genotype }\end{array}$} & $\begin{array}{l}\text { Number } \\
\text { of } \\
\text { samples }\end{array}$ & \multicolumn{3}{c}{ Band of GPDH activity } \\
\cline { 3 - 5 } & Samplerodimer & F1 \\
\hline$S / S$ & 8 & $29 \cdot 8 \pm 1 \cdot 6$ & $53 \cdot 1 \pm 2 \cdot 3$ & $17 \cdot 1 \pm 0 \cdot 9$ \\
$T 198 / H 31$ & 7 & $23 \cdot 8 \pm 2 \cdot 4$ & $59 \cdot 1 \pm 3 \cdot 4$ & $17 \cdot 1 \pm 2 \cdot 0$ \\
$S / H 31$ & 8 & $67 \cdot 2 \pm 1 \cdot 6$ & $32 \cdot 8 \pm 1 \cdot 1$ & n.d. \\
$F / T 198$ & 8 & $2.8 \pm 1.7$ & $37 \cdot 5 \pm 1.2$ & $59 \cdot 7 \pm 1 \cdot 8$ \\
\hline
\end{tabular}

n.d. $=$ non-detected.

the level of the enzyme produced by either $H 31$ or $T 198$.

As heterozygotes between $T 198, H 31, F$, or $S$ and $D f(2 L) G d h A$ each have half the diploid level of activity and CRM the phenomenon is not due to some compensatory mechanism mediated via promoters of different strengths (as have been found to modify alpha-amylase levels in mice; Schibler et al., 1983).

In summary, the results show that there is an interaction between elements close to the $G p d h^{+}$ locus in H31 or T198 and the normal Gpdh gene region, which enhances GPDH activity. The modification appears to be trans-acting and results in dominance in the level of enzyme activity and CRM.
We do not yet know the mechanism of this differential gene expression. The increase in GPDH activity could be due to increased transcription from the normal allele or increased in vivo stability of the enzyme produced. There is evidence of both kinds of modification of GPDH levels in D. melanogaster.

An EMS-induced $G p d h$ allele (BO-1-5) exists which has some of the characteristics of $T 198$ and H31 (O'Brien and MacIntyre, 1972). BO-1$5 / D f(2 L) G d h A$ heterozygotes have 12 per cent of normal diploid activity, but when $B O-1-5$ is in combination with standard alleles there are normal GPDH levels. Thus $B O-1-5$ fails to exhibit dosage compensation. O'Brien and MacIntyre argued that $B O-1-5$ is a point mutation which restricts the formation of homodimers but not of heterodimers in combination with the product of normal Gpdh alleles. Consistent with this explanation was the evidence that $B O-1-5$ showed interallelic complementation with two other EMS mutants. Kotarski et al., (1983) report a similar example in which another EMS induced mutant allele $\left(G p d h^{n S P 15}\right)$ has a trans effect on standard $G p d h$ alleles. In heterozygotes between either $S$ or $F$ and $G p d h^{n S P 15}$ the GPDH-F or GPDH-S homodimer is reduced in activity. They suggest that the nSP15 subunit binds with very high affinity to the GPDH$\mathrm{F}$ or $\mathrm{S}$ monomer to form a heterodimer with no enzymatic activity. However, mechanisms of these kinds would not fit our data as the increased GPDH activity in heterozygotes between either $T 198$ or $H 31$ and normal alleles does not appear to be due to increased heterodimer activities.

Natural populations contain modifiers which reduce the activity of GPDH by changing the rate of synthesis of the enzyme (Bewley, 1981 and Shaffer and Bewley, 1983). The modifiers act as cis-regulators and are closely linked to the $G p d h^{+}$ locus. A trans-acting dominant modifier, which acts by lowering GPDH degradation rates resulting in an increase in relative stability, has been described by King and McDonald (1983). However, this modifier is located on the third chromosome, whereas the structural gene $G p d h^{+}$ is on the second chromosome. The mechanism by which the in vivo stability of GPDH is increased in this case has not been described, but it is observed in the thorax and not in the abdomen. It is also known that the modifier has similar effects on the level of another abdominal protein. We are investigating whether $T 198$ or $H 31$ affect other pròteins.

Genetic variation in eukaryotes producing regulatory effects similar to those we have found 
may act via post-translational modification (Paigen, 1979). It will be interesting to test whether the trans-regulatory effect in heterozygotes between $H 31$ and $T 198$ and standard alleles is via transcriptional (Nevins, 1983) or post-translational mechanisms.

It is possible that $T 198$ and $H 31$ are structural variants at the $G p d h^{+}$locus, as mutations at the structural gene are known to alter the intracellular degradation rate of GPDH, and to give rise to different steady state levels (Bewley et al., 1980). However there is strong evidence that GPDH activity in D. melanogaster is controlled by a complex of regulatory and structural components which segregate as a unit (Wilkins, Shaffer and Bewley, 1982; Shaffer and Bewley, 1983) and the factor which distinguishes $T 198$ and $H 31$ from the standard alleles might be located within this complex. Genetic mapping of the H31 and T198 variants should provide information useful in future investigations of their DNA sequences. It is already clear that any modifier is effectively linked to the structural gene locus so that the electrophoretic phenotypes of $H 31 / S$ and $T 198 / F$ heterozygotes are excellent population markers for an unusual regulatory phenomenon.

Acknowledgements We wish to thank Doug Anderson, Bernard John, George Miklos and John Pateman, F.R.S. for helpful discussions about our data and comments on the manuscript.

\section{REFERENCES}

BeWley, G. C., DE ZURIK, J. M. AND PAGelson, G. 1980. Analysis of L-glycerol-3-phosphate dehydrogenase mutants in Drosophila melanogaster: complementation for intracellular degradation of the mutant polypeptide. Molec. gen. Genet., 178, 301-308.

BEWLEY, G. C. 1978. Heat stability studies at the $\alpha$-glycerolphosphate dehydrogenase locus in populations of Drosophila melanogaster. Biochem. Genet., 16, 767-775.

BEWLEY, G. C. 1981. Genetic control of the developmental program of L-glycerol-3-phosphate dehydrogenase isozy. mes in Drosophila melanogaster: identification of a cisacting temporal element affecting GPDH-3 expression. Dev. Genet., 2, 113-129.

BEWLEY, G. C., RAWLS, J. M. Jr. AND LUCCHESI, J. C. 1974. $\alpha$-Glycerolphosphate dehydrogenase in Drosophila melanogaster: kinetic differences and developmental differentiation of the larval and adult isozymes. J. Insect. Physiol, 20, 153-165.

CASTRO-SIERRA, E. AND OHNO, S. 1968. Allelic inhibition at the autosomally inherited gene locus for liver alcohol dehydrogenase in chicken-quail hybrids. Biochem. Genet., 1, 323-335.

CHAO, S. E. AND SCANDALIOS, J. G. 1971. Alpha-amylase of maize: differential allelic expression at the amy-1 gene locus, and some physiochemical properties of the isozymes. Genetics, 69, 47-61.
DICKINSON, W. J., ROWAN, R. G. AND BRENNAN, M. D. 1984. Regulatory gene evolution: adaptive differences in expression of alcohol dehydrogenase in Drosophila melanogaster and Drosophila simulans. Heredity, 52, 215225.

ENDO, T. 1971. Expression of allelic peroxidase isozymes in heterozygotes of Oryza perennis. Japan J. Genetics., 46, 1-5.

FINCHAM, J. R. S. 1966. Genetic complementation. W. A. Benjamin Inc., New York.

FREETH, A. L. AND GIBSON, J. B. 1985. Alcohol dehydrogenase and sn-glycerol-3-phosphate dehydrogenase null activity alleles in natural populations of Drosophila melanogaster. Heredity 55, 369-374.

GIBSON, J. B., WILKS, A. V., CAO, A. AND FREETH, A. L. 1985. Dominance for enzyme activity in Drosophila melanogaster. Experientia (In press).

GRELL, E. H. 1967. Electrophoretic variants of $\alpha$-glycerophosphate dehydrogenase in Drosophila melanogaster. Science, 158, 1319-1320.

HERRERA, R. J. 1979. Preferential gene expression of an amylase allele in interspecific hybrids of Xiphophorus (Pisces: Poeciliidae), Biochem. Genet., 17, 223-227.

KING, J. J. AND McDONALD, J. F. 1983. Genetic localization and biochemical characterization of a trans-acting regulatory effect in Drosophila. Genetics, 105, 55-69.

KLOSE, J. AND WOLF, U. 1970. Transitional hemizygosity of the maternally derived alleles at the $6 P G D$ locus during early development of the cyprinid fish Rutilys ratilus. Biochem. Genet., 4, 87-92.

KOTARSKI, M. A., PICKERT, S. AND MacINTYRE, R. J. 1983. A cytogenetic analysis of the chromosomal region surrounding the $\alpha$-glycerophosphate dehydrogenase locus of Drosophila melanogaster. Genetics, 105, 371-386.

KOTARSKI, M. A., PICKERT, S., LEONARD, D. A., LA ROSA, G. T. AND MacINTYRE, R. J. 1983. The characterization of $\alpha$-glycerophosphate dehydrogenase mutants in Drosophila melanogaster. Genetics, 105, 387-407.

LEWIS, N. AND GIBSON, J. 1978. Variation in amount of enzyme protein in natural populations. Biochem. Genet., 16, 159 . 170.

LINDSLEY, D. L. AND GRELL, E. H. 1968. Genetic variations of Drosophila melanogaster. Carn. Inst. Wash. Publ., Number 627.

MCCARRON, M., O'DONNELL, J., CHOVNICK, A., BHULlAR, B. S., HEWITT, J. AND CANDIDO, E. P. M. 1979. Organisation of the rosy locus in Drosophila melanogaster: further evidence in support of a cis-acting control element adjacent to the xanthine dehydrogenase structural element. Genetics, 91, 275-293.

NEVINS, J. R. 1983. The pathway of eukaryotic mRNA formation. Ann. Rev. Biochem., 52, 441-466.

NIESEL, D. W., BEWLEY, G. C., MILleR, S. G. AND ARMSTRONG, F. B. 1980. Purification and structural analysis of the soluble sn-glycerol-3-phosphate dehydrogenase isozymes in Drosophila melanogaster. J. Biol. Chem., 255, 4073-4080.

NIESEL, D. W., PAN, Y. E., BEWLEY, G. C., ARMSTRONG, F. B. AND LI, S. 1982. Structural analysis of adult and larval isozymes of sn-glycerol-3-phosphate dehydrogenase of Drosophila melanogaster. J. Biol. Chem., 257, 979-983.

O'BRIEN, S. J. AND MacINTYRE, R. J. 1972, The $\alpha$-glycerophosphate cycle in Drosophila melanogaster. II. Genetic aspects. Genetics, 71, 127-138.

PAIGEN, K. 1979. Genetic factors in developmental regulation. In Scandalios J. (ed.). Physiological Ecology. Academic Press, New York, pp. 1-49. 
PROCUNIER, J. D. AND TARTOF, K. D. 1978. A genetic locus having trans and contiguous cis functions that control the disproportionate replication of ribosomal RNA genes in Drosophila melanogster. Genetics, 88, 67-69.

SCHIBLER, U., HAGENBUCHLE, O., WELlbAUER, P. K. AND PITTET, A. C. 1983. Two promoters of different strengths control the transcription of the mouse alpha-amylase gene $A m y-1^{9}$ in the parotid gland and the liver. Cell, 33, 501-508.

SCHOLL, A. AND ANDERS, F. 1973. Tissue-specific preferential expression of the Xiphophorus xiphidium allele for 6-phosphogluconate dehydrogenase in interspecific hybrids of platy-fish (Poeciliidae, Teleosti). In Schroder, J. H. (ed.) Genetics and Mutagenesis of Fish. Springer-Verlag, New York, pp. 301-313.
SCHWARTZ, D. 1971. Genetic control of alcohol dehy. drogenase-a competition model for regulation of gene action. Genetics, 67, 411-425.

SHAFFER, J. B. AND BEWLEY, G. C. 1983. Genetic determination of sn-glycerol-3-phosphate dehydrogenase synthesis in Drosophila melanogaster. J. Biol. Chem., 258, 10027-10033.

WHITT, G. S. 1981. Evolution of isozyme loci and their differential regulation. In Scudder, G. G. E. and Reveal, J. L. (eds.). Evolution Today. Proc. 2nd Int. Cong. Syst. Evol. Biol., pp. 271-289.

WRIGHT, D. A. AND MOYER, F. H. 1966. Parental influences on lactate dehydrogenase in the early development of hybrid frogs in the genus Rana. J. Exp. Zool, 163, 215-230. 